\title{
Mesoscopic relaxation time of dynamic image correlation spectroscopy
}

\author{
Kyongok Kang \\ Forschungszentrum Juelich, Weiche Materie (Soft Matter), Juelich, Germany. \\ Email: k.kang@,fz-juelich.de
}

Received 16 February 2010; revised 20 March 2010; accepted 28 March 2010.

\begin{abstract}
Dynamical images contain useful information of how the objects behave in time and space. When the system is in biological fluids, the motion of the object is much over-damped; the relaxation time is the characteristics in a diffusive time scale. We have found dynamical states of melting and forming of small nematic domains $(10-30 \mu \mathrm{m})$ that are exhibited in the suspensions of fd-viruses under applied AC electric field amplitude at low frequency. Dynamic image correlation function is used for extracting the mesoscopic relaxation times of the dynamical states, which can be employed as an application to other dynamic imaging process of biologically relevant soft condensed matter and biomedical systems.
\end{abstract}

Keywords: Relaxation Time; Image Correlation Spectroscopy; Dynamical states

\section{INTRODUCTION}

Dynamic contrast-enhanced MRI (magnetic resonance imaging) is widely used for early detection of breast tumors, where the kinetics of signal variation are monitored after an injection of a paramagnetic contrast medium to distinguish between benign and malignant breast lesions [1]. The studies of microvasculature and microcirculation are useful in the sense that the solid tumor objects can disturb the regional blood flow, and influences the lack of linkage in a basement membrane. It is still a challenge to generalize the estimation of a regional blood flow and capillary permeability as volume fraction of the intravascular flow in breast tumors [1]. According to the authors in [1], the important criterions of reliable signal contrast (or relaxation times) are dependent on two assumptions; one is a negligible relaxavity of protons in different tissue compartment, and the other is much faster diffusion of water within a tissue and the exchange rate between them. The relaxation of bulk magnetization can be found and described by a single relaxation time [2]. These assumptions are feasible for a typical diffusion coefficient for water in soft tissues, and its relevant mean-squared displacement is reported as typical value of 30-60 $\mu \mathrm{m}$ size within about $125 \mathrm{~ms}$ recovery time [2]. Depending on the biomedical system, for instance, gray and white matter in the brain, heart, and kidney are in a relatively slow relaxation process (relaxation time as about $1 / \mathrm{s}$ ) within tissue and blood stream, as compared to other systems as liver and spleen [2]. Thus as far as the contrast agent is efficiently fast enough, then the slow process of water permeability can be negligible in terms of the exchange rates between different compartments for early detection of malignant tumor cells. Thus for an intermediate and slow time process, the single exponent time constant is convenient to characterize most biological relevant experimental parameters.

When the time-evolutionary information is engaged with a local space, it is somewhat complicated to distinguish between the time, and space-variable. For the system that contains self-mimicking sizes or shapes of objects (or images), but with different temporal events, it is extremely useful to predict what will happen after a certain time steps of events. Relatively large-time scale, such as astronomical or geographical-time, and macroscopic length are less relevant to predict "probable" events in mesoscopic time scale. Most physiochemical environments, i.e., medical, biochemical and biophysical issues are more engaged with a shorter-time interval. In the latter case, the system of interested objects is governed by thermodynamics and hydrodynamics, commonly in between the microscopic and macroscopic approach. As far as in time-correlation aspect, this mesos-copic time scale can be influenced by globally metastable but partially (or locally) in non-equilibrium states. Thus timecorrelation is still a challenge in mesoscopic length and time scale and in the speculation, nevertheless which is ambient in most soft matters. The demanding task of predicting "forward" temporal events relies on the complexity of dynamical entities (or inhomogenieties) themselves that evolve in the physiological situations (or en- 
vironments). Based on an appropriate statistical sampling, one can identify the quantitatively possible "observable" physiological objects at a time. Further estimation of the next-time expected value can be governed by the relaxation rate of the dynamical variables. Recently, a luminescent conjugate polyelectrolyte (LCP) is used for monitoring the amyloid plaques that can develop at various stages in human brain as known for Alzheimer's disease [3]. To diagnose Alzheimer's disease in the earlier stage, image color maps can be monitored as a non-invasive method. Image correlation spectroscopy is also used for protein dynamics on the cell surface and live-cell imaging to extract the information of aggregation and number density [4]. The desirable feature of the imaging correlation spectroscopy is suggested to achieve the diffusion coefficients and velocity for probing proteins in living cells [5]. Employing the medical imaging for the kinetic pathways of decaying or growing of bad cellular propagation is remained as a highly valuable issue. Much efficient and conclusive biomedical performance is needed to characterize the "urgency" in a simple and an "integrated" manner in time-resolving frames. Mesoscopic time and length scale imaging correlation spectroscopy could have its applications within biomedical technology of soft-tissues. The main difficulties to overcome will probably be related to small optical density differences. In case of soft-organs or tissues that consist of fibrous structures in the presence of multivalent ionic liquids, structures can be imaged through the birefringent nature of the fibrous structures. Then the orientation contrast can be conveniently visualized between crossed polarizers, which can be combined with timelapsed imaging processing.

We employ charged fibrous viruses (fd) suspensions to explore low frequency electric field-induced phase transitions due to their anisotropic conformations (or configurations) under optical axis. Especially, the partial ordering is found as increased with increasing field amplitude $[6,7]$. This kind of fibrous structure can be also found in other biological materials, such as in optical nerve cells, living polymers of F-actin and myosin fibers. Particularly optical nerve cells are directly engaged with our human brain "cognitive" central nervous system. Interesting, it is already known fact that there are object recognition activities with low frequency oscillations (called Gamma band, $\sim 40 \mathrm{~Hz}$ ) in human brain system $[8,9]$. It is the purpose of this paper that we introduce our working system of dynamic image correlation spectroscopy to predict the mesoscopic relaxation time of dynamical states of small nematic domains $(\sim 10-30 \mu \mathrm{m}$ size) that consist of charged fd-viruses particles.

Part of this research was supported by the EU-FP7 Network "NanoDirect" (contract number CP-FP-213948-2).

\section{METHOD AND DATA ANALYSIS}

\subsection{Sample Preparation}

Sample preparation is done by following the standard biological protocols [10] to prepare the fd-virus suspensions, from the XL1blue strain of E. coli as the host bacteria. The virus particles are purified by repeated centrifugation $10^{5} \mathrm{~g}$ for 5 hours and re-dispersed in Tris/ $\mathrm{HCl}$ buffers with varying concentration, depending on the final ionic strength. Bacteriophage fd is a rod-like macromolecule with a length of $880 \mathrm{~nm}$, a bare diameter of $\mathrm{D}=6.6 \mathrm{~nm}$, a persistence length at high salt concentration of $\mathrm{P}=2200 \mathrm{~nm}$, and a molecular weight of M.W. $\sim 1.64 \times 10^{7} \mathrm{~g} / \mathrm{mol}$. Much detailed information of molecular structures and dynamics of fd coat proteins are discussed in earlier times by means of scanning transmission electron microscope and solid-state NMR method [11-14]. Hydrodynamic properties and structure of fdviruses are measured by transient electric birefringence, and low-angle intensity fluctuations spectroscopy [15].

We prepare fd-virus suspensions at a given low ionic strength by osmotic equilibration (or dialysis) for 2 days with an analytical concentration of $1.6 \mathrm{mM}$ ionic strength of Tris/HCl buffer. The same buffer is used to dilute the suspensions (by typically $20 \%$ ) to the desired fd concentration. The ionic strength of the osmotic reservoirs is the ionic strength from which the electrostatic Debye-Huekel screening length is calculated. Furthermore, osmotic equilibration assures that the $\mathrm{pH}$ of the suspensions is equal to those of the osmotic reservoir. The Tris/HCl buffers are prepared by adding a small volume of $\mathrm{HCl}$ with a concentration of $1 \mathrm{M}$ to a $20 \mathrm{mM}$ TRIS solution till a $\mathrm{pH}=8.2$ is attained. This buffer is then diluted with deionized water for the preparation of the lower Tris/HCl-buffer concentrations. For buffer concentrations less than about $10 \mathrm{mM}$, both the ionic strength and the $\mathrm{pH}$ are affected by carbon dioxide that dissolves from the air, which in turn affects the surface charges of the fd-virus particles. A detailed account of the effect of dissolved carbon dioxide on the ionic strength and $\mathrm{pH}$ as a function of the buffer concentration can be found in [16]. Then the fd-concentration is measured by UV spectrometer to read optical density, or the extinction coefficient at approximately $269 \mathrm{~nm}$ wavelength.

The charge distribution on fd-virus particles has been interpreted on the basis of different models for an fdvirus particle from titration curves. The best fit to titration curves is found when assuming that the charges are located only on the hydrophilic outer region of adsorbed coat proteins, and not on the DNA strand of fd itself [17]. The iso-electric point of native $\mathrm{fd}$ is equal to $\mathrm{pH} \sim 4.2$. The surface charge is measured approximately negatively charged as $-9000 \mathrm{e}$ at $\mathrm{pH}=6.9$. The buffer capac- 
ity of the low-ionic strength Tris/ $\mathrm{HCl}$ buffer that we used (for $0.16 \mathrm{mM}$ salt concentration) is still sufficient to keep the $\mathrm{pH}>6.5$ [16], despite dissolving carbon dioxide from the air.

\subsection{Collection of Raw Image Intensity at Finite Time Steps}

The realization of morphological changes in local events at a space is much sensitive when the system is governed by dynamically variable quantities (or objects). As far as the system maintains a uniquely defined phase or state for much long-time as compared to the resolution of appropriate time binning, a decent characteristic time can be extracted for their relaxation behaviors. Also the optimization of AOI (area of interests) averaging is relevant to obtain "ensemble" average correlations, which influences the good average of background in statistics at far later time after the event occurs. Images are collected with an appropriate finite time step to develop the evolution of spatio-temporal morphological changes. Depending on the nature of dynamical states in the physical system, we have found that the resolution of time binning in sampling has to be optimized such that at least 3-10 times smaller than the actual time of dynamical events. For example, the dynamical state itself evolves in few tens of second, then appropriate time binning should be treated within a second as a time step.

Intensity-time traces for dynamical images are then constructed from the intensity map in 2-dimensional area, which normally depends on the optimization between the quality of the CCD camera and area of interest (AOI) in system. Figure 1 shows the example of collections of dynamical images at few seconds of time steps. Here the sampling time is every $1 \mathrm{sec}$, and total duration time is 1800 sec. The dynamical morphologies are driven by external AC electric field at a low frequency (of $10 \mathrm{~Hz}$ ) and amplitude (of $3.2 \mathrm{~V} / \mathrm{mm}$ ). This dynamical state is still slowly changing in time and space, so-called slow dynamical state as $D_{S}$ state. The change of visual observation of these slow dynamical states is typically occurred within $30 \mathrm{sec}$. By applying higher field amplitude, the slow dynamical state, $D_{S}$ transits to the fast dynamical state, i.e., $D_{f}$, where we have chosen multi-runs short time collections, as time binning as $300 \mathrm{~ms}$ and duration time for $60 \mathrm{~s}$ and 5 runs averaging. Due to the speed limitation of our camera, normally the camera setting ought to be optimized between fast speed and high reso lution option. We have optimized the CCD camera set as fast camera exposure time since the dynamical state domains are quite visible in $\mathrm{CCD}$ pixel by pixel.

We are then able to capture the dynamical events at least 3 or 4 different time steps within a sec temporal event in the fast dynamical, $D_{f}$-state.

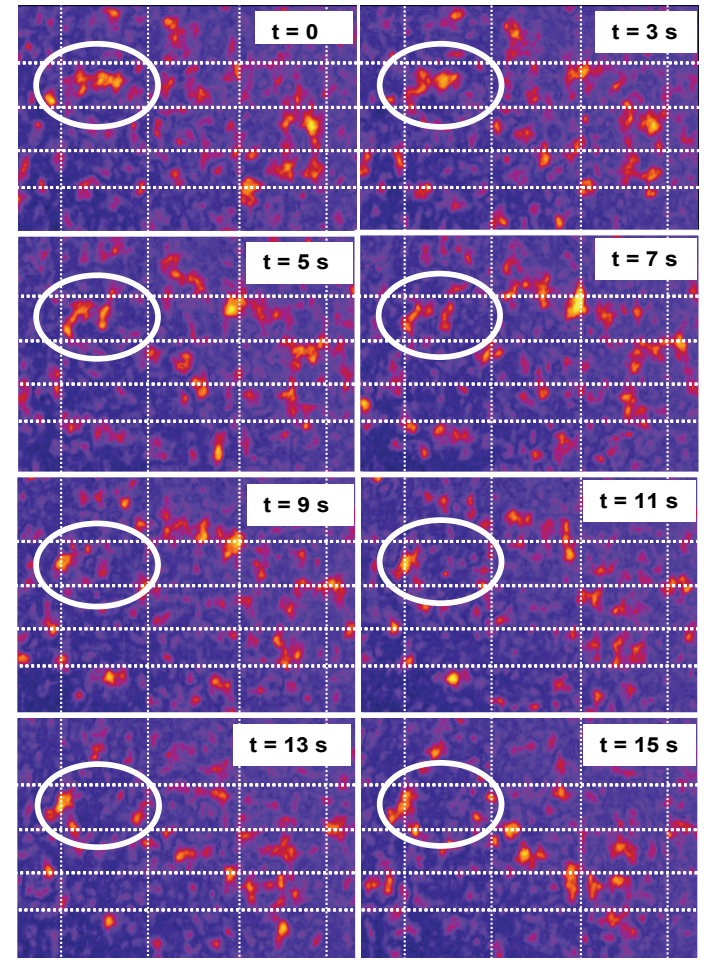

Figure 1. Temporal images of dynamical states that are induced by external electric AC field amplitude (of $3.2 \mathrm{~V} / \mathrm{mm}$ ) at a low frequency (of $10 \mathrm{~Hz}$ ). Field of view is of $400 \mu \mathrm{m} \times 300$ $\mu \mathrm{m}$, and the dotted lines are guiding eyes for detecting small neamtic domains of fd-virus particles. White circle is presented for tracing particular region of spatiotemporal images for dynamical states in each frame. Dynamical image correlation function is calculated as a resolution of single pixel averaging in 2-d over $1800 \mathrm{~s}$ duration time, where the flexible time resolution can be adapted as $0.3 \mathrm{~s}, 1 \mathrm{~s}$, for fats and slow dynamical states, respectively.

It turned out that this time binning may not be fast enough to characterize "completely" in the fast dynamical states, where the relaxation behaviors could be realized at much microscopic time scale. As a consequence, the background value turned out to be occasionally below zero, indicating that the time step is too large to carry on the local events correlations. It would be desirable when the time binning of the sampling time has to be tested with the background value to present as the dynamical state. Also it has to be chosen as much faster exposure $\mathrm{CCD}$ camera to ensure the exposure time of camera setting is much faster than the actual duration time for measurement. Thus by allowing the time step is much smaller than the change of visual morphological changes in dynamical states, time-lapsed images are recorded within the chosen frame setting, and saved (or exported) as time-sequential bmp files with 8 bit gray data that is mostly common format in handling image objects. 


\subsection{Construction of Modified Intensity Matrix}

To construct the intensity-time traces of images, the intensity matrix values of every pixel AOI $(1$ pixel $\times 1$ pixel) are reconstructed for dynamic image correlation function, as a "readable" Ascii format to correlate with different time-frames of AOI. The modified intensity matrix is defined as the subtracted intensity average value of AOI pixel for every time frame from the raw intensity data. Figure 2(b) shows the simple sketch of the enhanced contrast between "bright" and "dark" intensity AOI pixels.

The modified intensity matrix is then reconstructed as a building block of individual time frame, such that the each time frame image is subtracted with the overall average intensity value of the initial time frame, as can be seen in Figure 2(b). Then enhanced contrast is sketched as a bright (white) and dark (or black) unit pixel area, against the mean average of a gray color code. In principle, for a long time of duration, the change of the dynamical events can be completely "independent", so that at far later time after the event occurs, the time-correlation of dynamical states is expected to be " non-correlated" at the end of time event. This is briefly depicted in the long time later after an event occurs in Figure 2(b), which has now shown flipped from "white" to "black" and "black" to "white" code.

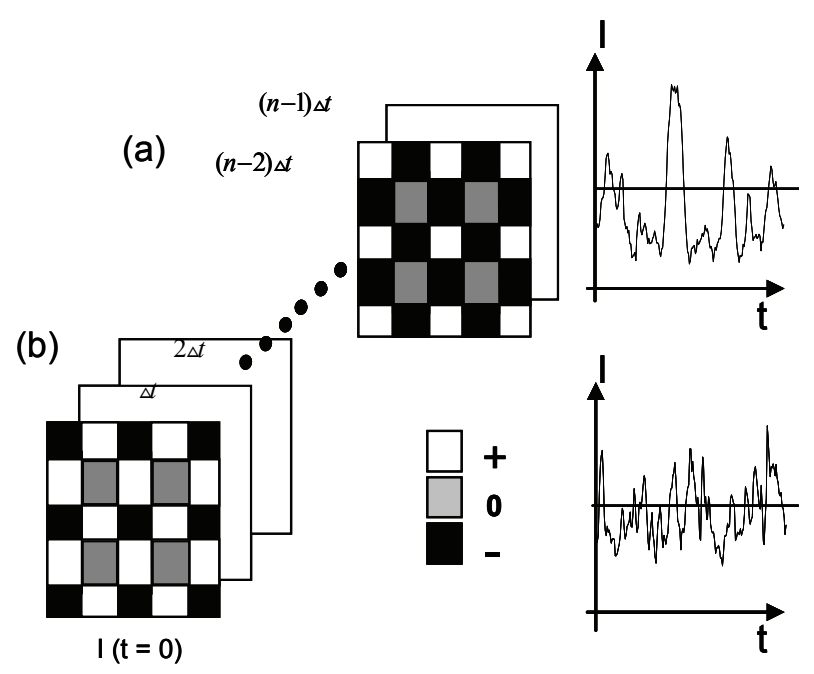

Figure 2. Simple sketches of the procedure of data analysis: (a) A time-lapsed sequence of raw images with a "readable" intensity matrix as 8 bit gray bitmap images. (b) A time-lapsed sequence of modified intensity matrix as a subtracted average intensity value at each time frame. Bright and dark intensity is contrasted in this modified intensity matrix. The resultant intensity traces over time is presented as typical slow and fast oscillations, and white, gray, and black unit cell represents the sign of the intensity values, notified in the modified intensity map in (b).
The right panels of Figure 2(b) are shown for the intensity - time traces for both slow and fast dynamical state, up and down figures, respectively. The apparent intensity oscillations are due to the spatio-temporal motion of dynamical states of the small nematic domains (of fd-virus particles).

\subsection{Calculation of the Image Correlation Function}

The correlation function is defined as

$$
C(t)=\frac{\langle[I(t)-<I(t)>][I(0)-<I(0)>]\rangle}{\left\langle[I(0)-<I(0)>]^{2}\right\rangle}
$$

where I is the transmitted intensity at a given pixel and the brackets $\langle\ldots\rangle$ denote averaging over all pixels. We made a program to calculate the dynamical image correlation function. We have found the best performance of the intensity averaging is 1 pixel by 1 pixel, as compared to 5 pixels by 5 pixels (or 10 pixels by 10 pixels) averaging. This may be related to a low magnification objective lens (10X objective) that is chosen for larger sampling of AOI in our system. Figure 3(a) shows the typical correlations functions of our dynamic imaging correlations as a function of increasing field amplitude (as indicated the arrow direction). Here, the relaxation time of dynamical states of melting and forming of small nematic-domains of fd-virus particles is presented as a function of applied field amplitude at a low frequency (of $10 \mathrm{~Hz}$ ).

\section{RESULTS AND DISCUSSION}

To briefly summarize the field-induced phase/state transitions of charged fibrous viruses (fd) at a low ionic strength:

- At low driving frequency and a low field amplitude, the chirality is induced, which is seen as a "stripe" texture (as a chiral nematic, $N^{*}$-phase) in depolarized light.

- At higher field amplitude, this field-induced chirality is slowly disconnected and developed as smaller domains that contain chiral nematics, so called as $N_{D}^{*}$ phase.

- Further increasing of the field amplitude, there is a discontinuous transition between melting away of chirality and appearing of small nematic domains that are kinetically responding in space and time. Thus this leads to the dynamical states as slow and fast in an increase of the amplitude, as $D_{S}$ and $D_{f}$-state, respectively.

Detailed electric phase/state diagrams are presented and characterized in [6].

As one can see in Figure 3, the relaxation time is decreased as increasing field amplitude; typical dynamical image correlation functions of a low frequency $(10 \mathrm{~Hz})$ are shown for varying the applied field amplitude 
$\mathrm{C}(\mathrm{t})$
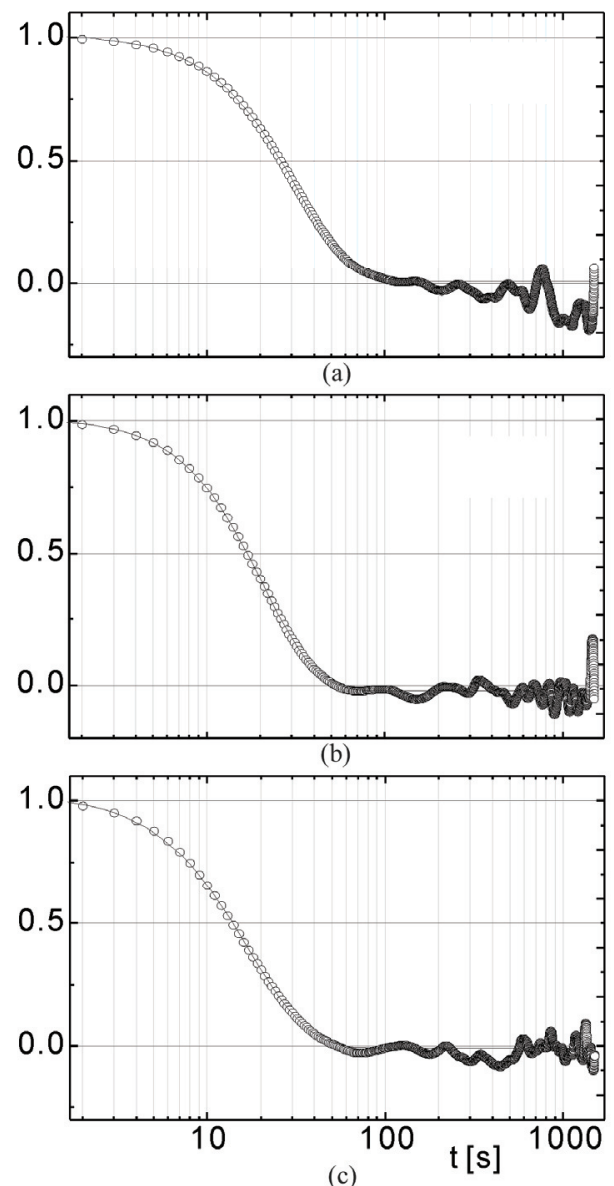

(c)

Figure 3. Typical dynamical image correlation functions of a low frequency $(10 \mathrm{~Hz})$ for a fd-concentration (of $2.8 \mathrm{mg} / \mathrm{ml}$ ): Relaxation time of dynamical states is decreased as an increase of the applied AC field amplitude as (a) $3.1 \mathrm{~V} / \mathrm{mm}$, (b) 3.5 $\mathrm{V} / \mathrm{mm}$, and (c) $3.9 \mathrm{~V} / \mathrm{mm}$. Note that there are relatively maintaining noise distribution in the background at longer time tails due to the disturbance of the external field.

from $3.1 \mathrm{~V} / \mathrm{mm}, 3.5 \mathrm{~V} / \mathrm{mm}$, and $3.9 \mathrm{~V} / \mathrm{mm}$. Also the microscopic discontinuity is observed in an increase of field amplitude at low frequency, at the transition of chiral-nematic phase to dynamical states (around 3.3 $\mathrm{V} / \mathrm{mm}$ ), by means of dynamic lights scattering [6,7]. The discontinuity may be due to the fact of "intrinsic" differences on the microscopic dynamics between chiralnemtic phase and non-chiral nematic domains that are present between isotropic and nematic coexistent phase. At this transition, the chirality slowly disappears and at the same time, non-chiral small nematic domains are becoming dynamic, in terms of their melting and formation of the small non-chiral nematic domains $(\sim 20 \mu \mathrm{m})$. Further increasing field amplitude at a low frequency, the dynamical states of melting and forming of small nematic domains are becoming faster. Thus kinetics of dynamical states is enhanced by an increase of field amplitude, as it can be seen in Figure 4(a). The mesoscopic relaxation times of these dynamical image correlation functions are indeed shown as "faster" as an increase of the field amplitude in Figure 4(b). These dynamic image correlation functions of applied field amplitude at a low frequency can be fitted with an exponential decay with a stretching exponent. The solid lines are fitted with a fitting function of

$$
C(t)=B+A \exp \left\{-(t / \tau)^{\beta}\right\}
$$

where $B=0, A \sim 0.5-0.6,0.5<\beta<1$, and $\boldsymbol{\tau}$ is the measure for a characteristic time for melting and appearing small nematic domains of fd-virus particles.

This characteristic time diverges on approach of the chiral-phase transition on lowering the field amplitude, in a mesoscopioc correlation time scale. Hoswever, the transition from the $D_{S^{-}}$to- $D_{f}$-state is defined as the point where the sharp decrease of $\boldsymbol{\tau}$ with increasing field amplitude ceases to occur. The mesoscopic relaxation time has a tendency of saturation that the dynamics be

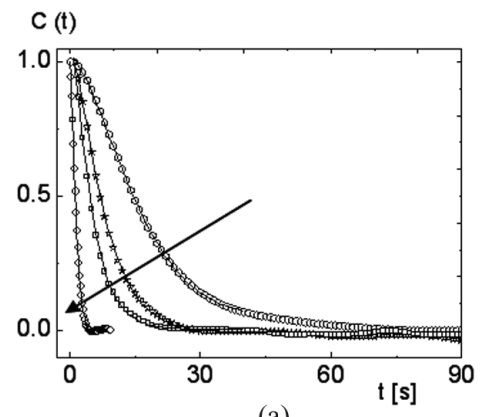

(a)

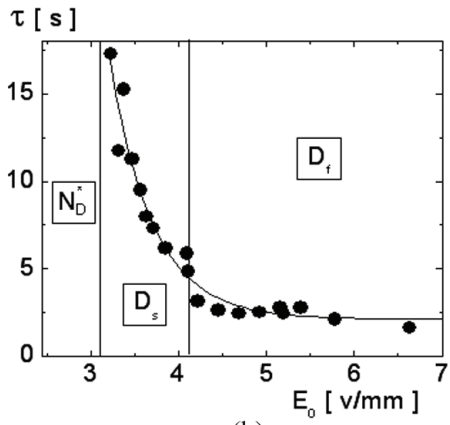

(b)

Figure 4. (a) Dynamical image correlation functions for various AC field amplitudes at low frequency (of $10 \mathrm{~Hz}$ ) of the fd-concentration $(2.8$ $\mathrm{mg} / \mathrm{ml}$ at $0.16 \mathrm{mM}$ salt). Arrow indicates the direction of an increase of field amplitude (from $3.26,3.78,4.09$, to $5.77 \mathrm{~V} / \mathrm{mm}$ ). Note that there is a very small modulation at longer time window; (b) Results of the mesoscopic relaxation time for dynamical image correlation functions for a low frequency (of $10 \mathrm{~Hz}$ ). 
comes essentially independent of the field amplitude. Whether this is due to the lack of our limited choice of time binning or the "intrinsic" feature of our dynamical system is not yet clear. We also have performed dynamic image correlation spectroscopy on approaching of the $D_{f}$ -to- $D_{S}$-state transition, by increasing frequency at a high field amplitude, where "critical" slowing down behaviors of small nematic domains are found, where the critical exponents can be obtained [18].

Figure 4(a) has shown the results of dynamical correlation functions for various field amplitudes, where the arrow indicates the increase of the field amplitude. Disregard of the background oscillations, the relaxation time decreases with an increase of the field amplitude. This means that the fast dynamical states are relaxing much faster than the slow dynamical states, as it is expected in the time correlation method. It also maintains as a "reversible", which is a good candidate for "tuning" the dynamical states with external driving frequency. Figure 4(b) is the resultant relaxation time as a function of applied field amplitude, where the field-induced $N_{D}^{*}$-phase, slow, $D_{S}$, and fast dynamical $D_{f}$ states are shown. Note that there are relatively "noisy" oscillations can be obtained in the long time tails (see Figure 4(a)), which may be due to the constant disturbance (or "feedback") of the external field. If the system is purely diffusive, then this correlation function has zero background. However, since the system is constantly feed backed by external alternating current amplitude, the intensity-time correlation function background does not go "directly" to absolute zero, but slightly oscillates around zero, although mathematically in principle should be "zero".

Thus we speculate these small contributions of white noise in terms of our low driving frequency as well. This can be useful for many biological imaging processing or biomedical electronics researches, since the signals are too much "weak" and "dark" to detect and to carry on in biological fluids and compartments (soft tissues).

Figure 5 indicates the zooming view of the background noise oscillations at larger time window for both slow (in (a)), and faster (in (b)) dynamical state. As one can see, the background oscillations are "modulated" in a complex manner, and the quality of the frequency is much "noisy" than the driving (or input) frequency.

In order to look at these possible oscillations in more detail, we propose a data analysis as follows. Prepare two independent backgrounds. One is from the measured back ground oscillation at larger times, as shown in Figure 6(a). The other is a "virtual" background oscillation (the sinusoidal waveform that is chosen as comparable amplitude in Figure 6(a) for a frequency that is $~$ $1 \%$ of driving frequency.

Typically $\sim 10 \%$ signal-to-noise ratio is feed into in

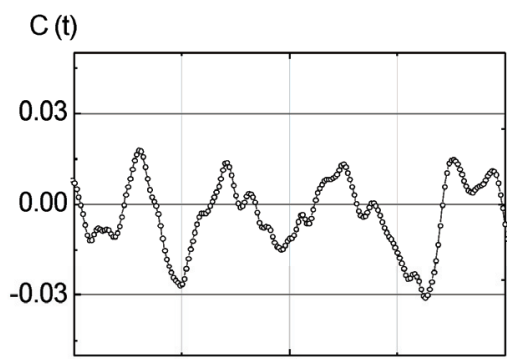

(a)

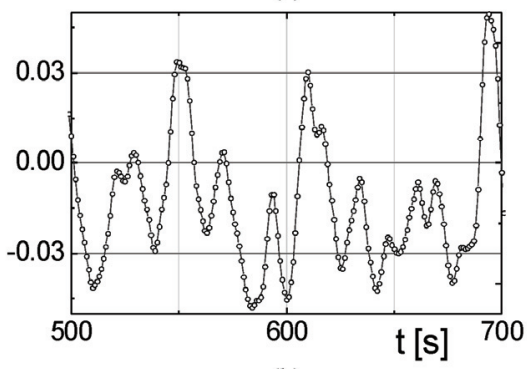

(b)

Figure 5. Zooming view of the background noise oscillation in the image correlation functions at longer time- window: (a) for slow dynamical state $(3.24 \mathrm{~V} / \mathrm{mm})$, and (b) for faster dynamical state $(3.86 \mathrm{~V} / \mathrm{mm})$.

the real correlation background noise. Then subtracted signals from the "virtual" oscillations are shown in Figure $\mathbf{6}(\mathrm{b})$, as given same time window, the resultant of the background signal is shown. The Fourier transform of the resulting signal gives the power spectrum given in Figure 6(c). Dashed line is the estimation of the power spectrum by using a frequency $(10 \mathrm{~Hz})$ pass filter. Interestingly, there is a sharp major peak value found as $1 \%$ of input driving frequency, which is good agreement of cut-off envelop signal. Whether this analysis is sufficient to contribute to the interpretation of the dynamics of the melting and forming of nematic domains needs further investigation. However, the weak background oscillations are not influenced by the choice of the temporalsensitivity, and indicate the intrinsic feature of fieldinduced dynamical states. It would be quite intriguing to know whether the smaller peaks in the power spectrum (between the major peaks) in Figure 6(c) can be amplified or modulated to tune the low frequency spectrum in the slowly varying dynamical systems. However this is beyond the scope of this paper.

\section{CONCLUSIONS}

For a dynamical image processing, we have used an experimentally working system for the time-lapsed dynamic image correlation spectroscopy. Series of collected time stacks of region of interests are chosen such that they maintain enough collective motion of single particles. In our experiment, typical average size of small nematic 

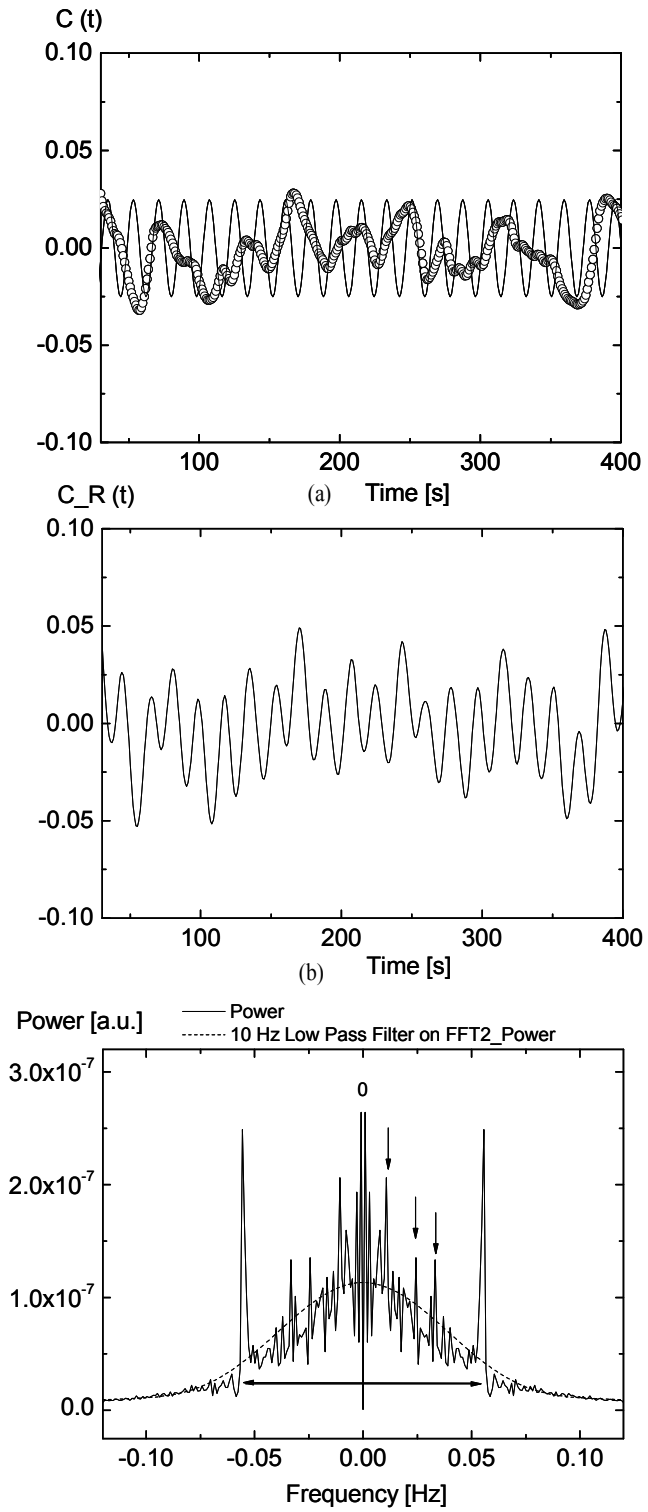

(c)

Figure 6. Further analysis of the background noise in the dynamical image correlation function: (a) Circle data points present a raw background signal, which is less than $5 \%$ of total $\mathrm{S} / \mathrm{N}$ ratio, and the thin solid line data is for an input signal with an optimized amplitude of an input frequency (of $10 \mathrm{~Hz}$ ). (b) Resultant of the background signal, defined as input signal in (b) subtracted from raw signal in (a). (c) FFT of the resultant signal in (c). Notice that small peaks are shown within between the centered beam and the major peak around $0.1 \mathrm{~Hz}$ (around $1 \%$ of dark signal). Thus the modulation in a background noise of a dynamical image correlation function can be used as additional information to characterize the dynamical states in much weak signal-oriented physical systems.

domain is approximately averaged value of $20 \mu \mathrm{m}$, where the dimensions of an individual fd-virus particle is about $\sim 1$ um long and its diameter is $\sim 0.03 \mu \mathrm{m}$ (for a low ionic strength). Thus the transmitted intensity at a given pixel, which is calibrated as $\sim 0.96 \mu \mathrm{m} /$ pixel conversion rate, is calculated for the image correlation function, as normalized with averaging over all pixels. The image speckle patterns of the dynamical states are obtained from the modified image sequence at a certain time step, subtracted from the raw image, and image correlation function with an initial intensity profile (at a time $\mathrm{t}=0 \mathrm{~s}$ ). It turned out to be important to choose the initial frame of the dynamical event as much as in quasi-equilibrium process to carry on the meso-scopic relaxation dynamics. For instance, we perform to recording after 3-5 min. of waiting after applying field condition to ensure that more or less the same dynamical events are occurred during the measurement. Also depending on the characters of the dynamical events, either slow or fast-process, the time binning should be at least $\sim 3-10$ times faster for extracting the appropriate time resolution. The resulting correlation function in time of the speckles (or our small nematic domains) is found to be as an exponential decay function with a slightly stretched exponent. The mesoscopic relaxation time of melting and forming of small nematic domains is then obtained systematically by varying the field amplitude at a low frequency. However, the mesoscopic relaxation time constant in "fast" dynamical states has tendency of saturation with the field amplitude at higher field amplitude. This may be related to either the lack of the resolution in our experiments, or the genuine behavior in the dynamical system. Thus it would be worthy to test the prediction of the relaxation time with an image correlation spectroscopy for other similar dynamical events of biological interest or medical environments.

Interestingly, we have also found that the time constant for the relaxation behavior diverges on approaching to the chiral-phase regime from the transition of dynamical states (close to $\sim 3.2 \mathrm{~V} / \mathrm{mm}$ ). This may imply the dynamical states are the consequence of the interaction of charged fd-rods via induced polarization effect in thick electric double-layers. We are currently developing the possible theory for an explanation.

To conclude, mesoscopic time and length scale imaging correlation spectroscopy needs a challenge to customize the biomedical technology of soft-tissues. The main difficulties are not only related to the density differences, but also the "weak" and "reliability" of signals/ images of temporal evolution in micro-structural contrast. However as far as any soft-organs or tissues consist of fibrous structures in the presence of multivalent ionic liquids, any types of the elastic envelops can be monitored by their orientations in biological fluids. Then the orientation contrast can be conveniently visualized by depolarized light that can be combined with time-lapsed 
imaging processing, as we have introduced in this report. If one could come up with an integrated package for fast time-lapsed imaging processing to pixel-to-pixel intensity auto correlation signal progressing, then this could be an application to characterize the mesoscopic relaxation behaviors of dynamical situations in many relevant soft biological and biomedical front matters. It can be adapted to early detection or "progressive" medicare issues. For instance, biological fluids (as blood, saliva, urine) can be accessed by in-vitro method under a low external electric field to recognize the "abnormality" related the growth/decay of "foreign" cells in terms of the change of microscopic "viscosity" or "resistance".

In this aspect, the "controllable" non-equilibrium approach of the dynamical image correlation spectroscopy is much more informative than a steady-state or quasiequilibrium image processing.

\section{ACKNOWLEDGEMENTS}

Author thanks to J.K.G. Dhont for his scientific insight and introduce the flexible-time resolving image correlation functions to characterize the relaxation time in slow and fast dynamical states.

\section{REFERENCES}

[1] Eason, G., Brix, G., Kiessling, F., Lucht, R., Darai, S., Wasser, K., Delorme, S. and Griebel, J. (2004) Microcirculation and microvasculature in breast tumors: $\mathrm{Ph}$ armacokinetic analysis of dynamic MR image series. Magnetic Resonance in Medicine, 52, 420-429.

[2] Donahue, K.M., Weisskoff, R.M. and Burstein, D. (1997) Water diffusion and exchange as they influence contrast enhancement. Journal of Magnetic Resonance Imaging, 7, 102-110.

[3] Peter, K., Nilsson, R., Aslund, A., Berg, I., Nystrom, S., Konradsson, P., Herland, A., Inganas, O., Stabo-Eeg, F., Lindgren, M., Westermark, G.T., Lannfelt, L., Nilsson, L.N.G. and Hammarstrom, P. (2007) Imaging distinct conformational states of amyloid- $\beta$ fibrils in Alzheimer's Diseases using novel luminescent probes. ACS Chemical Biology, 2, 553-560.

[4] Wiseman, P. and Peterson, N.O. (1999) Image correlation spectroscopy II, optimization for ultrasensitive detection of preexisting platelet-derived growth factor$\beta$ receptor oligomers on intact cells. Biophysical Journal, 76, 963-977.

[5] Costantino, S., Comeau, J.W.D., Kolin, D.L. and Wisemann, P. (2005) Accuracy and dynamic range of spatial image correlation and cross-correlation spectroscopy. Biophysical Journal, 89, 1251-1260.

[6] Kang, K. and Dhont, J.K.G. (2010) Electric-field induced transitions in suspensions of charged colloidal rods. Soft Matter, 6, 273-286.

[7] Kang, K. (2009) Image and signal correlation of electricfield induced charged fibrous viruses (fd). Proceedings of the $31^{\text {st }}$ Annual International Conference of the IEEE Engineering in Medicine and Biology Society, San Francisco, 1294-1297.

[8] Csibra, G., Davis, G., Spratling, M.W. and Johnson, M.H. (2000) Gamma oscillations and object processing in the infant brain. Science, 290(5496), 1582-1585.

[9] Baudry, C. and Bertrand, T.O. (1999) Oscillatory gamma activity in humans and its roles in object representation. Trends in Cognitive Sciences, 3(4), 151-162.

[10] Sambrook, J., Fritch, E.F. and Maniatis, T. (1989) Molecular cloning: A laboratory manual. Cold Spring Harbor, New York.

[11] Lipka, J.J., Lippard, S.J. and Wall, J.S. (1979) Visualization of polymercurimethane-labeled fd-bacteriophage in the scanning transmission electron microscope. Science, 206(4425), 1419-1421.

[12] Cross, T.A. and Opella, S.J. (1979) NMR of fd coat protein. Journal of Supramolecular Structure, 11(2), 139-145.

[13] Gall, G.M., Cross, T.A., Diverdi, J.A. and Opella, S.J. (1982) Protein dynamics by solid state NMR: Aromatic rings of the coat protein in $\mathrm{fd}$ bacteriophase. Proceedings of the National Academy of Sciences, 79(1), 101-105.

[14] Schwind, P., Kramer, H., Kremser, A., Ramsberger, U. and Rasched, I. (1992) Subtilisin removes the surface layer of the phage fd coat. European Journal of Biochemistry, 210(2), 431-436.

[15] Newman, J., Swinney, H.L. and Day, L.A. (1977) Hydrodynamic properties and structure of fd-virus. Journal of Molecular Biology, 116(3), pp. 593-606.

[16] Kang, K., Wilk, A., Patkowski, A. and Dhont, J.K.G. (2007) Diffusion of spheres in isotropic and nematic networks of rods: Electrostatic interactions and hydrodynamic screening. Journal of Chemical Physics, 126(21), 214501-(1-17).

[17] Zimmermann, K., Hagedorn, J. Heuck, C.C., Hinrichsen, M. and Ludwig, J. (1986) The ionic properties of the filamentous bacteriophage Pf1 and fd. The Journal of Biological Chemistry, 261, 1653-1655.

[18] Kang, K. and Dhont, J.K.G. (2009) Criticality in a nonequilibrium, driven system: Charged colloidal rods (fdviruses) in electric fields. The European Physical Journal E, 30(3), 333-340. 